\title{
Digital design and parameters optimization for plasmonic circuits
}

\author{
Alexander Shesterikov, Andrey Leksin, and Alexei Prokhorov* \\ Vladimir State University named after A. G. and N. G. Stoletovs, 600000 Vladimir, Russia
}

\begin{abstract}
The mathematical models for the CAD-platform of plasmonic circuits design have been developed. This platform provides the efficient framework for computer-aided design of semiconductor quantum dots and full-field electromagnetic simulation of surface plasmon-polariton propagation in plasmonic waveguides. The topology of an all-plasmonic devices based on graphene layers and quantum dots is proposed for the first time.
\end{abstract}

The main purpose of this work is the development and software implementation of mathematical models and numerical algorithms as the basis for creating an Computer-Aided Design Platform (CAD) to design the plasmonic circuits. Such software should include the calculation of characteristics for signal processing centers, as well as the design of waveguide schemes for signal transmission. Here, the carriers of information are the surface plasmonpolaritons (SPPs) generated near the interface boundary of materials with different types of conductivity. Such perspective materials as graphene, doped graphene [1], boron nitride, and others were chosen as the basis for design of proposed system. The semiconductor non- and core-shell quantum dots (QDs) based on A3B5 and A2B6 semiconductors [2] were selected as the signal processing centers. Using dimensional effects, i.e. dependences of frequencies, polarizations and dipole moments of electron transitions on size of QD, should allow to design the gates and triggers corresponding to different schemes of SPP-QD interaction.

At the first stage, we created the database containing information about the characteristics for used semiconductor compounds, such as the energy of the valence band ceiling, the bottom of conduction band, the width of forbidden band, as well as the effective masses of electron and hole. Using this data, we developed the mathematical methods allowing the calculation of the dependences of electron levels positions, as well as the frequencies, dipole moments and relaxation rates for the transitions between these levels on the size of QD. Based on the developed mathematical models, the module for automated calculation of the parameters of semiconductor QD was created and tested. We also implemented the finite difference time domain method (FDTD [3]) to simulate the propagation of SPP in plasmonic waveguides based on perspective materials. We have developed a mathematical model for the full-field electromagnetic simulation in graphene nanostructures. This module allows to design an arbitrary graphene-like layout for for SPP propagation control. In the process of

\footnotetext{
* Corresponding author: avprokhorov33@mail.ru
} 
module testing on standard layouts, we calibrated our results based on results obtained by other authors $[4,5]$.

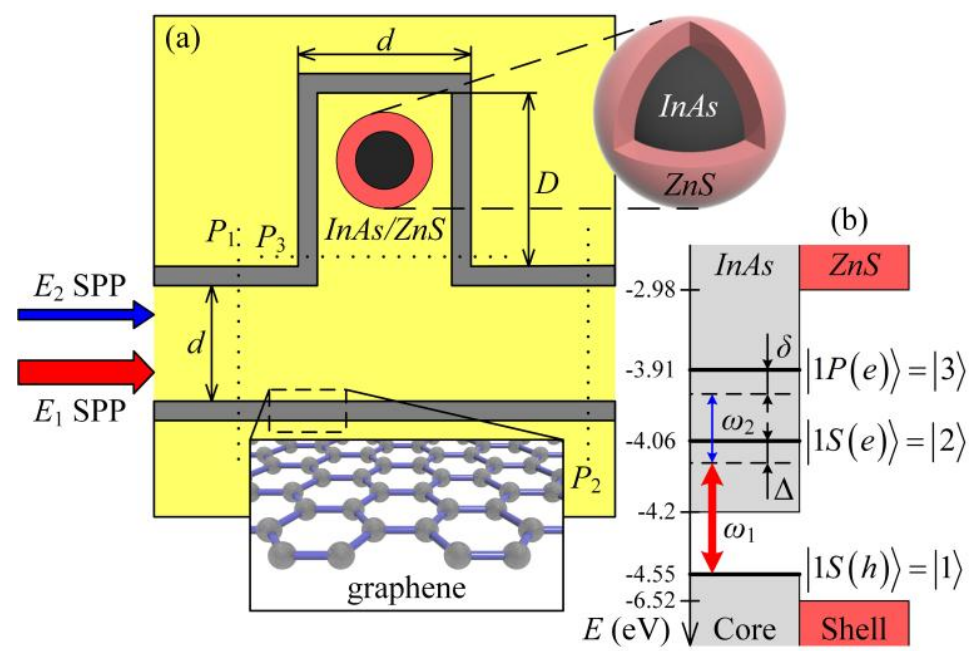

Fig. 1. (a) The model of graphene waveguide integrated with stub nanoresonator loaded with core-shell QD. (b) Relative position between energy gaps and band offsets of InAs-ZnS bulk semiconductors; the Ladder-type interaction scheme of two SPP modes in InAs/ZnS QD.

The developed platform was the basis for designing the all-plasmonic transistor. Such a transistor is designed on the basis of a $20 \mathrm{~nm}$ bilayer graphene waveguide integrated with 25 $\mathrm{nm}$ height stub-nanoresonator and loaded with semiconductor core-shell InAs/ZnS quantum dot, see Fig.1a. The device is based on the use of a strong SPP-QD interaction. The key idea is to obtain the "pi" phase shift of signal SPP due to the strong non-linear cross-interaction with the pump SPP in condition of the Ladder-type scheme of QD-SPP interaction, see Fig.1b. The control of signal SPP phase shift via the pump SPP intensity allows as to switch from destructive to constructive interference in the stub and change the signal SPP transmitance from a few percent to 90 percent.

This work was supported by the Foundation for Assistance to Small Innovative Enterprises, Agreement 2226GS1/37022 (START-1).

\section{References}

1. M. Aliofkhazraei , N. Ali, W. I. Milne, C. S. Ozkan, S. Mitura, J. L. Gervasoni Graphene science handbook. Electrical and Optical Properties (London: CRC Press, Taylor \& Francis Group, 2016).

2. A. V. Fedorov, A. V. Baranov, I. D. Rukhlenko, T. S. Perova, K. Berwick, Phys. Rev. B. 76, 045332 (2007).

3. D. M. Sullivan, Electromagnetic simulation using the FDTD method ( New York: Wiley-IEEE Press, 2000).

4. B. Wang, X. Zhang, X. Yuan, J. Teng, Appl. Phys. Lett. 100, 131111 (2012).

5. P. C. Sarker, Md. M. Rana, A. K. Sarkar, Optik 144, 1 (2017). 